\title{
APLICAÇÃo DE Zr/Ti-PILC NO PROCESSO DE ADSORÇÃO DE Cu(II), Co(II) E Ni(II) UTILIZANDO MODELOS FÍSICO-QUÍMICOS DE ADSORÇÃO E TERMODINÂMICA DO PROCESSO
}

\author{
Denis Lima Guerra* e Claudio Airoldi \\ Departamento de Química Inorgânica, Instituto de Química, Universidade Estadual de Campinas, CP 6154, \\ 13084-971 Campinas - SP, Brasil \\ Vanda Porpino Lemos e Rômulo Simões Angélica \\ Departamento de Geoquímica, Centro de Geociências, Universidade Federal do Pará, Rua Augusto Corrêa, s/n, \\ 66075-110 Belém - PA, Brasil

\section{Rúbia Ribeiro Viana} \\ Departamento de Recursos Minerais, Instituto de Ciências Exatas e da Terra, Universidade Federal do Mato Grosso, \\ Av. Fernando Corrêa da Costa, s/n, 78068-900 Cuiabá - MT, Brasil
}

\begin{abstract}
APPLICATION OF Zr/Ti-PILC IN THE ADSORPTION PROCESS OF Cu(II), Co(II) and Ni(II) USING ADSORPTION PHYSICOCHEMICAL MODELS AND THERMODINAMICS OF THE PROCESS. The aim of this investigation is to study how Zr/Ti-PILC adsorbs metals. The physico-chemical proprieties of $\mathrm{Zr} / \mathrm{Ti}$-PILC have been optimized with pillarization processes and $\mathrm{Cu}$ (II), $\mathrm{Ni}$ (II) and $\mathrm{Co}$ (II) adsorption from aqueous solution has been carried out, with maximum adsorption values of $8.85,8.30$ and $7.78 \times 10^{-1}$ $\mathrm{mmol} \mathrm{g}^{-1}$, respectively. The Langmuir, Freundlich and Temkin adsorption isotherm models have been applied to fit the experimental data with a linear regression process. The energetic effect caused by metal interaction was determined through calorimetric titration at the solid-liquid interface and gave a net thermal effect that enabled the calculation of the exothermic values and the equilibrium constant.
\end{abstract}

Keywords: adsorption isotherms; metals; $\mathrm{Zr} / \mathrm{Ti}$-pillared clay.

\section{INTRODUÇÃO}

A adsorção de metais por superfícies minerais é um processo reconhecidamente importante que controla a biodisponibilidade dos metais em ambientes aquáticos ${ }^{1-9}$. O tratamento clássico de efluentes contendo metais envolve processos físico-químicos de precipitação, troca iônica, adsorção e extração por solventes, sendo mais utilizado o de precipitação, que é inadequado no tratamento de grandes volumes de efluentes, devido à baixa eficiência e elevados custos ${ }^{10}$. Neste caso, é mais recomendado o uso de recursos naturais sem tratamento prévio, como solos argilosos ferruginosos ricos em substâncias húmicas ${ }^{11} \mathrm{e}$, também, materiais naturais quimicamente modificados, como exemplos zeólitas naturais impregnadas com materiais magnéticos ${ }^{12,13}$ e argilas modificadas por processo de pilarização ou intercalação. Tais materiais se enquadram no grupo de materiais porosos, desenvolvidos pela engenharia de materiais, com ampla aplicação em catálise heterogênea ${ }^{14}$ e como adsorventes ${ }^{15-21}$.

Com a evolução das pesquisas em busca de novos materiais que fossem utilizados como adsorventes, surgiram os materiais meso e microporosos, em particular as zeólitas ou peneiras moleculares, que atuam efetivamente com alto poder adsortivo, provocado por suas propriedades texturais. No decorrer do avanço das pesquisas surgiram as argilas pilarizadas com diversos polioxicátions, que produziram resultados satisfatórios em processos de adsorção. Vários metais pesados foram testados em meio líquido com adsorventes minerais quimicamente modificados, sendo que os resultados obtidos mostraram que as argilas pilarizadas são bastante eficientes em processos de adsorção $0^{9-13}$. Diversos estudos comprovam a eficiência das argilas em processos adsortivos e catalíticos, sendo que as argilas mais comumente empregadas em estudos de adsorção

*e-mail:dlguerra@iqm.unicamp.br pertencem ao grupo da esmectita, principalmente a montmorilonita $^{14-18}$.

Estudos de adsorção de cobre realizados com amostras de esmectitas pilarizadas com pilares mistos de zircônio e titânio mostram um aumento do poder de adsorção em relação à natural, com variação das capacidades de retenção catiônica entre 0,10 e 0,45 mmol g ${ }^{-1}$, sendo, portanto, comparável com a capacidade obtida pelas zeólitas ${ }^{18-28}$. A acidez de uma série de cátions governa o perfil da isoterma de adsorção, a seqüência de seletividade e a energética de interação adsorvente/adsorbato ${ }^{11,14,18,19}$.

Amostras de bentonita, tanto natural como pilarizada, mostraram-se eficientes na remoção de cromo (III) de águas residuais de curtumes; verificou-se que quando pilarizada com titânico, a capacidade de troca é maior que a natural, com redução de 1850 para 25 ppm do caráter ácido ${ }^{29}$. A melhor viabilidade de remoção foi verificada com a investigação utilizando água residual em pH 5,0. Alguns estudos também foram realizados visando a retenção do cátion amônio existente em águas residuais e efluentes de fábricas de tintas, tendo como adsorvente as esmectitas pilarizadas com titânio ${ }^{29,30}$.

A aplicação de esmectitas pilarizadas como adsorventes é sugerida, por estes materiais apresentarem pilares que mantêm separadas as camadas da estrutura da argila e propiciam que as dimensões de suas cavidades se tornem bem superiores às das zeólitas $^{31-46}$. Neste trabalho foi feita uma avaliação sobre o uso de esmectita pilarizada na adsorção de $\mathrm{Cu}(\mathrm{II}), \mathrm{Ni}(\mathrm{II})$ e $\mathrm{Co}(\mathrm{II})$, sendo selecionada uma amostra de esmectita da região noroeste do estado do Pará, que foi submetida à pilarização mista com poliidroxicátions de zircônio e titânio. Este estudo objetivou investigar a eficiência desta matriz pilarizada, na adsorção destes metais e empregar os modelos de linearização de Langmuir, Freundlich, Temkin para ajustar os dados experimentais aos modelos de 
isotermas e à termodinâmica do processo de interação metal/Zr/Ti -PILC na interface sólido/líquido, com a determinação das grandezas termodinâmicas através de estudo calorimétrico.

\section{PARTE EXPERIMENTAL}

\section{Esmectita original}

A amostra natural de esmectita empregada neste estudo pertence a um perfil laterítico, proveniente da Serra de Maicuru, situada no município de Monte Alegre, Pará, Brasil. Tem capacidade de troca catiônica (CTC) igual a 5,8 $\mathrm{mmol} \mathrm{g}^{-1}$ de argila.

\section{Preparação da esmectita original}

A separação granulométrica foi realizada com a amostra devidamente pesada em balança analítica (Bosch P115), dispersa em água destilada e submetida a um aparelho de ultrasom (Thorton), com o objetivo de provocar maior dispersão das partículas e sua homogeneização. A amostra foi tratada com $\mathrm{Na}_{2} \mathrm{P}_{2} \mathrm{O}_{7}$ para evitar a floculação e depois de separada por centrifugação (Heraeus Christ GMBH Osterade), utilizando velocidade e tempo de separação da fração silte de $1000 \mathrm{rpm}$ e $2 \mathrm{~min}$, respectivamente, novamente a fração argila resultante foi centrifugada (2500 rpm e $10 \mathrm{~min}$ ) para que se tivesse a retirada do $\mathrm{Na}_{2} \mathrm{P}_{2} \mathrm{O}_{7}$, conforme recomendação ${ }^{16,20,21,24}$.

$200,0 \mathrm{mg}$ da fração da amostra de esmectita original foram pesadas em uma balança analítica, dispersas em água destilada em balão volumétrico com capacidade de $10,0 \mathrm{~cm}^{3}$. Pequenas alíquotas foram retiradas e dispersas em lâminas de vidro, previamente identificadas, até seu completo preenchimento. As lâminas contendo as alíquotas foram secas ao ar em temperatura ambiente durante um período de 3 dias, sobre uma bancada ${ }^{20,21}$.

\section{Preparação da solução pilarizante}

O etóxido de titânio preparado foi tratado com uma solução de $\mathrm{HCl}$, a fim de se obter razão molar $\mathrm{HCl} / \mathrm{Ti}$ variando de 1 a 4 . Nesse típico procedimento foram usadas soluções de $\mathrm{Ti}\left(\mathrm{OC}_{2} \mathrm{H}_{3}\right)_{4}(5,0 \mathrm{~mol}$ $\left.\mathrm{dm}^{-3}\right)$ e $\mathrm{HCl}\left(0,10 \mathrm{~mol} \mathrm{dm}^{-3}\right)$ Aldrich $\circledast$, sendo que a solução final sofreu um envelhecimento à temperatura ambiente por $3 \mathrm{~h}$, para resultar no polioxicátion de titânio $\mathrm{TiO}_{\mathrm{x}}(\mathrm{OH})_{2 \mathrm{x}} \operatorname{com} \mathrm{x}=0,12^{15,18}$. A intercalação da esmectita com polioxicátion de Ti foi efetuada com razão molar $\left[\mathrm{H}^{+}\right] /\left[\mathrm{Ti}^{4+}\right]=0,24$.

A solução pilarizante de zircônio, $\left[\left(\mathrm{Zr}(\mathrm{OH})_{2} \cdot 4 \mathrm{H}_{2} \mathrm{O}\right)_{4}\right]^{8+}$, foi obtida a partir do método bem estabelecido ${ }^{6}$, através da reação do acetato de zircônio $\mathrm{Zr}\left(\mathrm{C}_{2} \mathrm{H}_{3} \mathrm{O}_{2}\right)_{4} \mathrm{H}_{2} \mathrm{O}\left(0,10 \mathrm{~mol} \mathrm{dm}^{-3}\right)$ Aldrich ${ }^{\circledR}$ diluído em ácido acético $\left(2,0 \mathrm{~mol} \mathrm{dm}^{-3}\right)$ para que se tivesse a razão molar $\mathrm{H}^{+} / \mathrm{Zr}$ $=1,5$, em agitação mecânica constante por um período de $2 \mathrm{~h}$, com um tempo de envelhecimento de $24 \mathrm{~h}$.

A solução contendo pilares mistos foi obtida utilizando as mesmas soluções descritas acima, da seguinte forma: a solução de titânio foi misturada lentamente sem variação de temperatura com a solução de zircônio em vigorosa agitação mecânica, por um período de $3 \mathrm{~h}$ na razão 1:1; após este período, a solução ficou em repouso por $12 \mathrm{~h}$ em temperatura ambiente ${ }^{6}$.

\section{Processo de pilarização}

Uma amostra da esmectita original foi preparada previamente em atmosfera de etilenoglicol em temperatura ambiente por um período de $24 \mathrm{~h}$, com o objetivo de expandir o espaço interlamelar e, com isso, facilitar a entrada dos íons intercalantes ( $\mathrm{Ti}, \mathrm{Zr}$ ). Posteriormente foram colocadas em agitação magnética com a so- lução pilarizante mista (mistura das soluções de Ti e $\mathrm{Zr}$ descritas no item anterior) por um período de $3 \mathrm{~h}$ em temperatura de 273,15 $\mathrm{K}$ (processo de intercalação). Após a intercalação, as amostras foram filtradas, lavadas, secadas a $333,15 \mathrm{~K}$, calcinadas em temperatura de $723,15 \mathrm{~K}$ e conservadas em dessecador por todo o período que compreende a utilização dos métodos de caracterização $0^{20,21}$.

\section{Métodos de caracterização}

Para a obtenção de difratogramas de difração de raios-X (DRX), foi utilizado o método do pó para as amostras natural e pilarizada; o equipamento utilizado é de marca Philips PW1050, controlado por um sistema PW 3710, com radiação KÅ do cobre $(1,54060 \alpha)$, monocromador e gerador de tensão e corrente para $45 \mathrm{KV}$. Corrente de $35 \mathrm{~mA}$ e passo de $1 \% / \theta / \mathrm{min}$.

As análises químicas das amostras natural e pilarizada consistiram primeiramente no processo de solubilização das mesmas, através de abertura ácida, seguida das determinações pelo método de absorção atômica com modelo Variam 220, na faixa entre 185-900 $\mathrm{nm}$, para os elementos $\mathrm{Na}, \mathrm{K}, \mathrm{Ca}, \mathrm{Al}$; para os elementos $\mathrm{Si}, \mathrm{Fe}, \mathrm{Ti}$ foi utilizado o método de espectroscopia na região do UV-visível, equipamento com duplo-feixe Varian modelo Cary 1E, utilizando celas de $10,0 \mathrm{~mm}$ de caminho óptico a uma faixa entre 360-830 $\mathrm{nm}, \mathrm{Fe}$ sob a forma do complexo ortofenantrolina e Ti como peróxido complexo. A solubilização total das amostras utilizadas seguiu o procedimento proposto ${ }^{47}$, consistindo na abertura ácida com $\mathrm{HF} / \mathrm{HNO}_{3} / \mathrm{HClO}_{4} / \mathrm{HCl}$.

As determinações da área específica (AE), volume total dos poros (VTP) e diâmetro médio dos poros (DMP) foram obtidas a partir de $0,249 \mathrm{~g}$ de cada amostra. As amostras natural e pilarizada foram aquecidas a uma temperatura de 423,15 K pelo período de $1 \mathrm{~h}$. Após o aquecimento, foram resfriadas à temperatura do nitrogênio líquido (77 K) em um analisador de adsorção gasosa Quantachrome/ nova-1200/5.01, realizando-se, assim, as análises que duraram $7 \mathrm{~h}$. Através dessas análises foram obtidas as respectivas isotermas de adsorção-dessorção. A partir dos dados dessas isotermas foram calculados os valores de AE e VP. A área especifica foi calculada pelo método BET (Brunauer-Emmett-Teller) e o volume total de poros calculado à pressão parcial $\left(\mathrm{p} / \mathrm{p}_{0}\right)$ de 0,998955 pelo método $\mathrm{BJH}$ (Barrett-Joyner-Halenda).

Para os estudos de microscopia eletrônica de varredura (MEV) as amostras natural e pilarizada foram dispersas em lâminas de vidro em temperatura de $298,15 \mathrm{~K}$ e metalizadas com ouro. Foram realizadas varreduras em todas as lâminas para a obtenção de imagens. O instrumento utilizado foi um modelo Leo- Zeiss, $430 \mathrm{Vp}$, em condições de análise utilizando imagens secundárias obtidas a $20 \mathrm{KV}$, com distância de trabalho de $11 \mathrm{~mm}$.

As investigações calorimétricas foram realizadas utilizando um calorímetro isotérmico da Thermometric, modelo LKB 2277. Foram pesadas diretamente na cela calorimétrica, em torno de 20,0 $\mathrm{mg}$ de esmectita nas formas natural e pilarizada. Adicionou-se, então, $2,0 \mathrm{~cm}^{3}$ de água desionizada e o vaso calorimétrico foi acoplado à torre do sistema, para finalmente ser inserida no canal do equipamento. O sistema é agitado vigorosamente e o controle de temperatura a 298,15 $\pm 0,20 \mathrm{~K}$. Após atingir a estabilidade térmica do sistema, volumes de $10,0 \mu \mathrm{L}$ da solução do metal são adicionados periodicamente, através de uma bomba injetora carregada com microsseringa de volume total $0,50 \mathrm{~cm}^{3}$.

\section{Procedimento e técnica utilizada para a determinação da adsorção dos metais}

Inicialmente foi feita investigação prévia para verificar qual o 
pH que melhor adsorveria os metais; a adsorção foi verificada em faixa de $\mathrm{pH} 1,0$ a 5,0. Todos os experimentos foram feitos em 298,15 $\pm 0,20 \mathrm{~K}$ e a variação de $\mathrm{pH}$ foi realizada através da adição de volumes de $\mathrm{NaOH}\left(0,10 \mathrm{mmol} \mathrm{\textrm {dm } ^ { - 3 }}\right)$ e $\mathrm{HNO}_{3}\left(0,10 \mathrm{mmol} \mathrm{dm}^{-3}\right)$ utilizando Systronic pH meter modelo $362 \mu$.

Os estudos de adsorção dos metais foram realizados em pH 5,0 e temperatura controlada em $298,15 \pm 0,20 \mathrm{~K}$. A capacidade de adsorção dos cátions divalentes por grama da matriz $\mathrm{N}_{\mathrm{f}}$, $\mathrm{Ni}$ (II) (nitrato de níquel, Vetec), $\mathrm{Cu}$ (II) (nitrato de cobre, Vetec), $\mathrm{Co}(\mathrm{II})$ (nitrato de cobalto, Vetec) pela argila natural e pilarizada foi investigada utilizando o método de batelada, em que amostras de $0,050 \mathrm{~g}$ de argila natural e pilarizada em $20,0 \mathrm{~cm}^{3}$ de água desionizada receberam alíquotas que variaram de 1,00 a $2,00 \mathrm{~cm}^{3}$ de uma solução de $0,050 \mathrm{~mol} \mathrm{dm}^{-3}$ de cátions, previamente padronizada. A quantificação foi feita através de ICP-OES em um equipamento Perkins Elmer 3000 DV, utilizando o sobrenadante de cada ponto. Para a leitura, os sobrenadantes sofreram diluições para adequar sua concentração à janela de leitura do equipamento. $\mathrm{O}$ valor de cada $\mathrm{N}_{\mathrm{f}}$ foi determinado pela Equação 1. O modelo utilizado na análise da isoterma construída por cada ponto foi o de Langmuir descrito pela Equação $2^{48}$.

$\mathrm{N}_{\mathrm{f}}=\left(\mathrm{N}_{\mathrm{ad}}-\mathrm{N}_{\mathrm{s}}\right) / \mathrm{m}$

$\mathrm{N}_{\mathrm{f}}$ é o número de moles fixos por grama de matriz, $\mathrm{N}_{\mathrm{ad}}$ é o número de moles adicionados inicialmente na matriz, $\mathrm{N}_{\mathrm{s}}$ é o número de moles encontrado no sobrenadante e número máximo de moles na formação da monocamada e m é a massa da matriz utilizada ${ }^{48}$.

$\left[\mathrm{C}_{\mathrm{s}} / \mathrm{N}_{\mathrm{f}}\right]=\left[1 /\left(\mathrm{K}_{\mathrm{L}} \mathrm{b}\right)\right]+\left[\mathrm{C}_{\mathrm{s}} / \mathrm{b}\right]$

$\mathrm{C}_{\mathrm{s}}$ é a concentração em mol dm ${ }^{-3}$ do sobrenadante em equilíbrio e b é uma constante de proporcionalidade que contempla a constante de equilíbrio $\mathrm{K}_{\mathrm{L}}$. Considerando $\mathrm{C}_{\mathrm{s}} / \mathrm{N}_{\mathrm{f}}$ como variável dependente $\mathrm{e}$ $\mathrm{C}_{\mathrm{s}}$ como variável independente, obtêm-se os valores de $\mathrm{K}_{\mathrm{L}}$ e b, em que $1 / \mathrm{K}_{\mathrm{L}}$ b é o coeficiente linear e $1 / \mathrm{b}$ é o coeficiente angular da reta $^{48}$.

A Equação de Freundlich é a seguinte ${ }^{4,50}$ :

$\mathrm{N}_{\mathrm{f}}=K f \mathrm{C}^{1 / n}$

em que $\mathrm{n}$ indica, quantitativamente, a reatividade dos sítios energéticos do material e $K f$ pode sugerir a adsorção do íon no material. Aplicando propriedades logarítmicas tem-se:

$\log N_{f}=\log K f+1 / n \log C$

Fazendo-se o gráfico de $\log \mathrm{N}_{\mathrm{f}}$ versus $\log \mathrm{C}$, obtêm-se os valores de $K f$ e n, sendo Log $K f$ o coeficiente linear e $1 /$ n o coeficiente angular da reta.

A Equação de Temkin é a seguinte:

$\mathrm{N}_{\mathrm{f}}=\mathrm{A} \operatorname{Ln} \mathrm{C}+\mathrm{B}$

A e B são parâmetros de equação. Fazendo-se o gráfico de $\mathrm{N}_{\mathrm{f}}$ versus Ln C, obtém-se os valores, sendo B o coeficiente linear e A o coe- ficiente angular da reta. Os coeficientes obtidos a partir destas equações foram utilizados para ajustar o modelo teórico aos dados experimentais.

\section{RESULTADOS E DISCUSSÃO}

\section{Caracterização da argila pilarizada}

Os resultados obtidos com a análise química comprovaram a existência de grande teor de ferro na amostra da esmectita original analisada, que foi observado na difração de raios X; além da presença de esmectita, também foi verificada a presença de picos característicos do mineral goethita $(\mathrm{FeO}(\mathrm{OH}))(0,43 \mathrm{~nm})$, hematita $\left(\mathrm{Fe}_{2} \mathrm{O}_{3}\right)(0,31 \mathrm{~nm})$, caulinita $\left(\mathrm{Al}_{2} \mathrm{Si}_{2} \mathrm{O}_{5}(\mathrm{OH})_{4}\right)(0,35 \mathrm{~nm})$ e quartzo $\left(\mathrm{SiO}_{2}\right)(0,44 \mathrm{~nm})$. Nesta amostra foi observado um percentual significativo de $\mathrm{Na}$, que pode ser apontado como contra íon (Tabela 1).

Os resultados encontrados na difração de raios-X para valores de $\mathrm{d}_{001}$ para as razões molares em estudo estão mostrados na Figura 1. São apresentados os difratogramas das amostras natural e pilarizada na razão de molar 2,0 e calcinação em 723,15 K, temperatura ideal para a calcinação. Foi verificado que o valor de $d_{001}$ sofreu alteração de 1,40 para 1,93 nm. Foi observado, também, que a intensidade e a largura do pico $\mathrm{d}_{001}$, alcançadas com a pilarização, não apresentaram grande alteração em comparação com o difratograma da matriz natural, sugerindo que não houve interferência significativa no grau de cristalinidade da matriz natural com a introdução dos íons de zircônio e titânio e com a submissão da matriz intercalada à temperatura de calcinação, 723,15 K. Fatos que comprovam a eficiência do método de pilarização com estes íons, apresentando inovação com a utilização do etilenoglicol como espaçador, favorecendo assim a entrada dos íons pilarizantes.

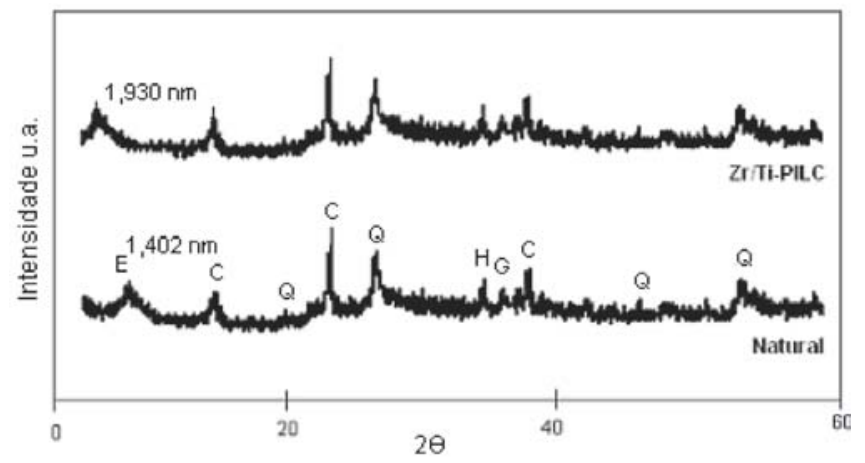

Figura 1. Difratogramas de raios- $x$ das amostras natural e pilarizada com pilares mistos (Zr e Ti): esmectita $(E)$, caulinita $(C)$, hematita $(H)$, goetita $(G)$ e quartzo $(Q)$

Esta modificação estrutural indica que a esmectita pilarizada tem possivelmente melhor poder reativo em processos de adsorção que aquela que está na forma natural. Um fato importante está no aumento considerável no valor de $\mathrm{d}_{001}$ que, conseqüentemente, sugere maior número de sítios reativos na estrutura desta matriz, fundamentando-se no fato de que os sítios, quando na forma natural, estavam em difícil

Tabela 1. Análise química mostrando a variação da composição na estrutura da esmectita, com o processo de pilarização

\begin{tabular}{lcccccccc}
\hline Amostra & $\% \mathrm{SiO}_{2}$ & $\% \mathrm{Al}_{2} \mathrm{O}_{3}$ & $\% \mathrm{FeO}$ & $\% \mathrm{ZrO}_{2}$ & $\% \mathrm{TiO}_{2}$ & $\% \mathrm{CaO}$ & $\% \mathrm{Na}_{2} \mathrm{O}$ & $\% \mathrm{~K}_{2} \mathrm{O}$ \\
\hline Natural & 59,30 & 13,40 & 13,91 & - & 3,58 & 3,78 & 2,20 & 3,80 \\
$\mathrm{Zr}$ Ti-PILC & 59,29 & 13,14 & 13,15 & 6,15 & 6,39 & 0,94 & 0,170 & 0,75 \\
\hline
\end{tabular}


acesso no interior da estrutura, mas com o processo de pilarização tornaram-se expostos e reativos, contribuindo assim com o poder de adsorção da matriz.

Na Tabela 1 estão apresentados os resultados da análise química das amostras natural e pilarizada, cujos resultados possibilitam verificar a quantidade de íons ( $\mathrm{Zr}$ e Ti) introduzidos na estrutura da esmectita. Observa-se que o valor de $\mathrm{Zr}$ e Ti aumentou com a introdução dos íons pilarizantes na estrutura da matriz, chegando a 26,9\% e os valores de $\mathrm{Ca}, \mathrm{Na}, \mathrm{K}$ decaíram, levando à conclusão que ocorreu a troca iônica destes íons localizados na região interlamelar com os íons intercalantes $\mathrm{Zr}$ e Ti. Foi verificada uma pequena alteração nos valores de $\mathrm{Si}$, aumento importante nos valores de Ti e aparecimento de teor significativo de potássio que pode estar associado à presença do íon na região interlamelar do mineral ilita, que apresenta naturalmente propriedades estruturais semelhantes às da mica e moscovita ${ }^{17}$. Comparando os valores da análise elementar para as amostras, natural e pilarizada, foi observada maior concentração de zircônio e titânio na matriz pilarizada (Zr/Ti-PILC); este resultado era esperado, pela existência dos pilares na estrutura da matriz pilarizada.

Na Tabela 2 estão listados os resultados da análise textural: área específica (AE); volume total de poros (VTP) e diâmetro médio dos poros (DMP). Foi comprovado que com a pilarização o valor da área específica cresceu atingindo o valor de $208,3 \mathrm{~m}^{2} \mathrm{~g}^{-1}$; analisando a isoterma de adsorção/dessorção de nitrogênio, formada para a amostra pilarizada, é observado que esta forma de isoterma pertence à classificação do tipo IV, segundo BDDT (Brunauer, Deming, Deming e Teller) ${ }^{20}$, conforme a Figura 2. Houve a formação de ciclo de histerese em altas pressões relativas na amostra pilarizada, característica dos materiais mesoporosos, podendo conter microporos. Um ligeiro afastamento de curva observado em baixas pressões, está relacionado com o fenômeno de expansão, que é característico de materiais cujo grau de expansão do sólido durante a adsorção depende da rigidez deste sólido ${ }^{22}$.

Tabela 2. Resultados da análise textural das esmectitas natural e pilarizada: $\mathrm{d}_{001}$ - espaçamento basal, AE- área especifica, VTP- volume total de poros, DMP- diâmetro médio dos poros

\begin{tabular}{lcccc}
\hline Amostra & $\mathrm{d}_{001}(\mathrm{~nm})$ & $\mathrm{AE}\left(\mathrm{m}^{2} / \mathrm{g}\right)$ & $\mathrm{VTP}\left(\mathrm{cm}^{3} / \mathrm{g}\right)$ & $\mathrm{DMP}(\mathrm{nm})$ \\
\hline Natural & 1.42 & 44,37 & 0,12 & 4.51 \\
Zr/Ti -PILC & 1.93 & 208,03 & 0,49 & 17.99 \\
\hline
\end{tabular}

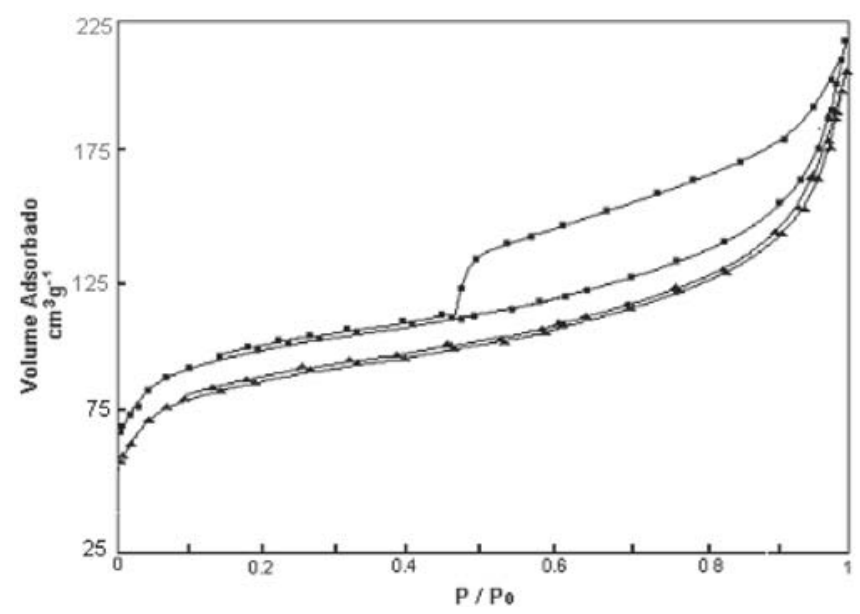

Figura 2. Isotermas de adsorção-dessorção das esmectitas natural ( $\mathbf{\Delta}) e$ pilarizada (
Com base nos resultados fornecidos pela microscopia eletrônica de varredura para a matriz pilarizada com os métodos em estudo (Figura 3), é possível afirmar que o processo de pilarização não produz nenhuma mudança na morfologia da esmectita, quando pilarizada com pilares mistos com o método de pilarização empregado, foi observado que em todos os experimentos a esmectita permaneceu na forma de grão primitivo, apresentando-se de forma foliada sugerindo a existência do mineral ilita nas extremidades, fato comprovado pela variação da composição química que foi observada pelos métodos de análise química descritos.

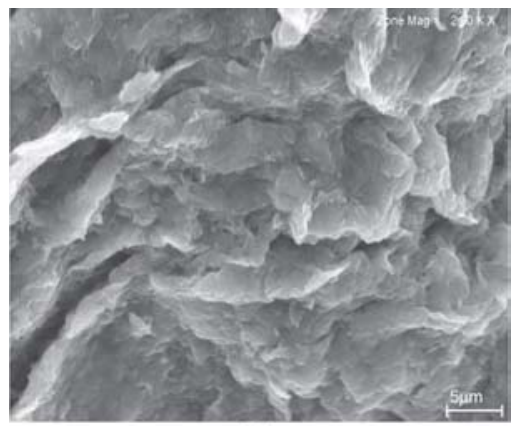

(a)

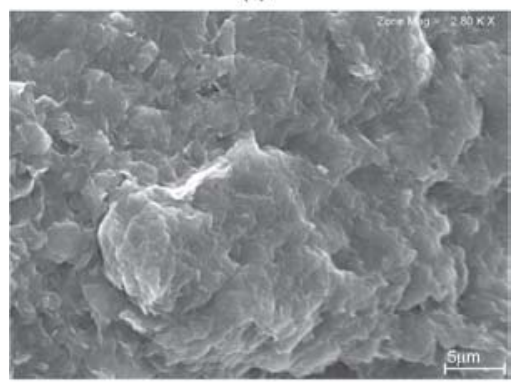

(b)

Figura 3. Imagens de microscopia eletrônica de varredura das argilas natural (a) e pilarizada $(\mathrm{Zr} / \mathrm{Ti}$-PILC) (b)

\section{Adsorção de metais}

Na Figura 4 estão os resultados obtidos com a investigação do $\mathrm{pH}$ ideal para a adsorção dos metais, na faixa de $\mathrm{pH}$ estudada (1,0$5,0)$. Foi observado que com o aumento do $\mathrm{pH}$, o valor de número de moles finais adsorvidos $\left(\mathrm{N}_{\mathrm{f}}\right)$ também aumentava; com esta verificação foi adotado o valor de 5,0 como $\mathrm{pH}$ ideal para o restante dos experimentos de adsorção e termodinâmica.

Nos processos de adsorção normalmente prima-se por escolher equações que tenham a característica de ser facilmente linearizáveis e, conseqüentemente, estimar os parâmetros graficamente, através de coeficientes angular e linear da reta resultante da linearização dos dados experimentais. Os modelos comumente empregados são Langmuir, Freundlich e Temkin, conforme mostram as Figuras 5 e 6.

A teoria de Langmuir fundamenta-se no fato da adsorção ocorrer em sítios uniformes, considera a ocorrência da adsorção em uma superfície homogênea contendo um número finito de sítios adsorventes com limitação adsortiva, em uma monocamada ${ }^{48}$. A afinidade iônica, independente da quantidade de material adsorvido, também considera que não há interação entre moléculas adsorvidas em sítios próximos ${ }^{48,49}$.

O modelo de Freundlich considera a não uniformidade das superfícies reais, corresponde a uma distribuição exponencial dos efeitos térmicos da reação de adsorção, quando aplicados a materiais porosos adsorvendo em meio líquido, descreve bem a adsorção iônica 
dentro de limites estabelecidos de concentração, mas em processos de adsorção em concentrações de valores maiores, esta equação apresenta limitações. Admitindo-se uma distribuição logaritmica de sítios ativos, que na verdade, constitui um tratamento válido quando não existe interação apreciável entre as moléculas de adsorbato ${ }^{49,50}$.

A teoria de Temkin parte do princípio que não existe diferen-ciação na estabilidade dos centros de adsorção que, no caso das matrizes pilarizadas, se considera na região externa dos pilares e nas superfícies internas que foram expostas com a pilarização. Outra consideração relevante é que os sítios mais energéticos são primeiramente ocupados pelos íons previamente em solução aquosa, estabelecendo uma relação de inversão proporcional entre afinidade eletrônica e adsorção $0^{50}$.

Comparando os resultados obtidos com as isotermas de adsorção mostrados na Figura 5, foi verificado que a adsorção de cobre foi maior na matriz pilarizada, atingindo a concentração de equilíbrio em $8,85 \mathrm{x}$ $10^{-1} \mathrm{mmol} \mathrm{g}^{-1}$; a adsorção de cobalto atingiu o valor de $8,30 \times 10^{-1}$ mmol $\mathrm{g}^{-1} \mathrm{e}$ a adsorção de níquel apresentou menor afinidade com a matriz pilarizada, alcançando o valor máximo de $7,78 \times 10^{-1} \mathrm{mmol} \mathrm{g}^{-1}$. A complexão de íons metálicos com sítios ativos imobilizados em superfícies externa e interna da estrutura esmectítica e parte exterior dos pilares é comandada pelo microambiente em torno destes sítios, geralmente centros básicos, ou seja, grupos hidroxilas que estão presentes na estrutura, como hidroxilas como assessórios presos aos pilares; hidroxilas ainda existentes nas superfícies, principalmente nas internas, onde a remoção da água é dificultada pela posição de folhas tetraédrica e octaédrica e, grupos hidroxilas remanescentes da estrutura primitiva da argila, presentes na região interlamelar. Levando a concluir que o processo de quimissorção é controlado pelo posicionamento e quantidade de agrupamentos hidroxilas em regiões periféricas, como
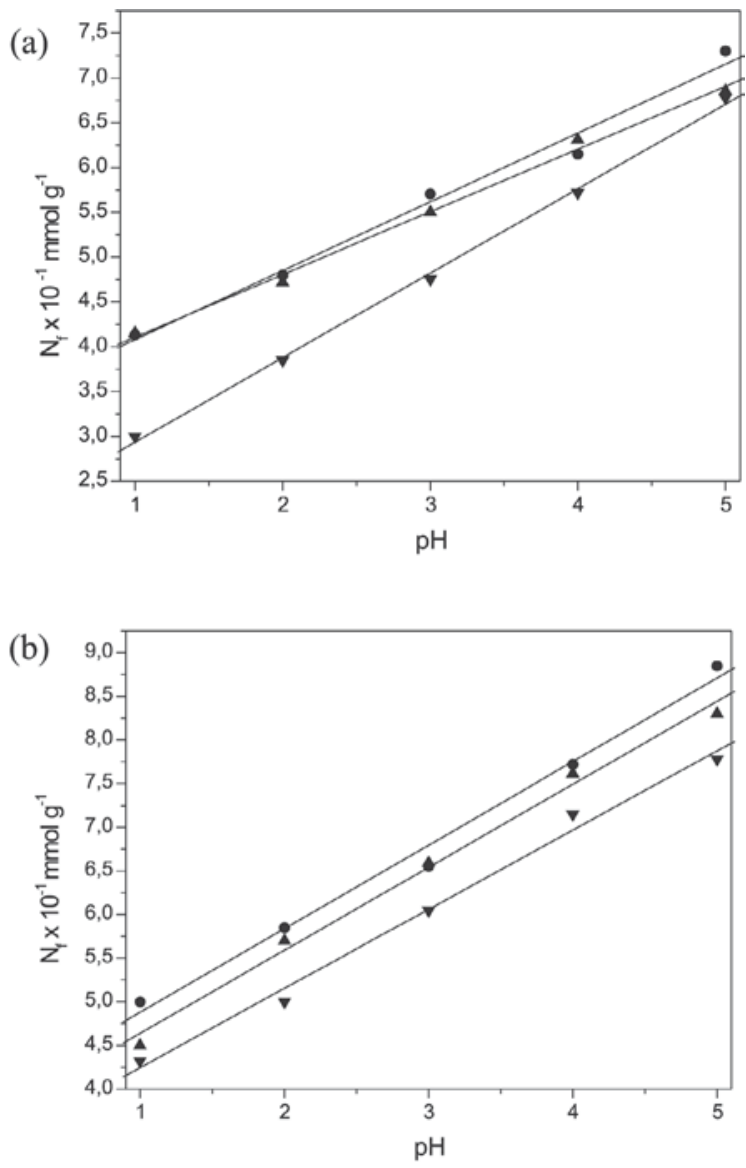

Figura 4. Estudo da variação do pH em adsorção dos metais em temperatura de 298,15 \pm 0,20 K: Níquel $(\boldsymbol{\nabla})$, Cobalto $(\mathbf{\Delta})$, Cobre $(\boldsymbol{\bullet})$
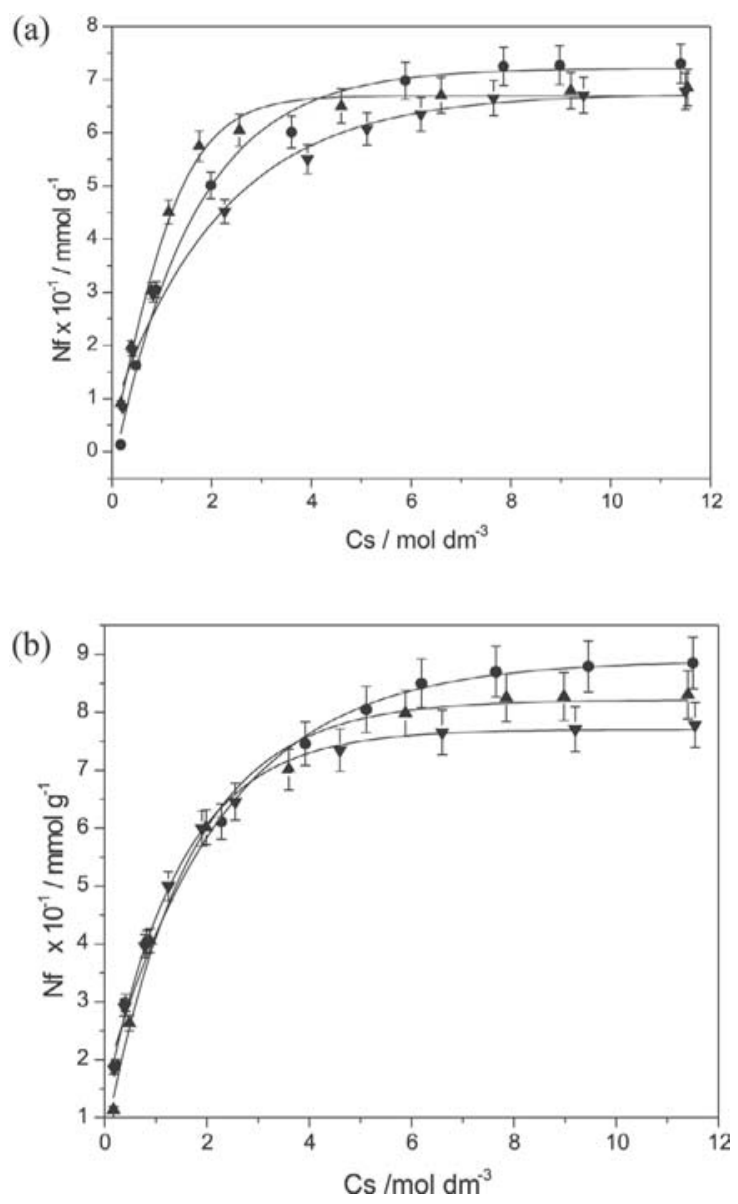

Figura 5. Isotermas de adsorção para os metais em estudo tendo como adsorvente ( $p H$ 5,0 e temperatura 298,15 $\pm 0,20 \mathrm{~K}$ ): esmectita natural (a) $e$ esmectita pilarizada $\mathrm{Zr} / \mathrm{Ti}$-PILC (b): Níquel $(\boldsymbol{\nabla})$, Cobalto $(\mathbf{\Delta})$, Cobre (

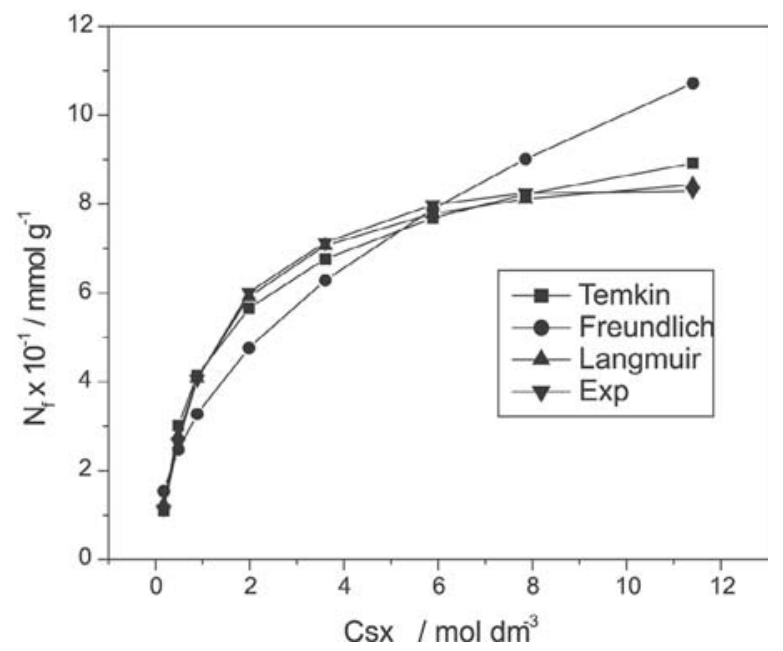

Figura 6. Exemplificação dos valores experimentais de adsorção, modelados com as equações de Langmuir, Freundlich, Temkin (pH 5,0 e temperatura $298,15 \pm 0,20 \mathrm{~K})$

nas bordas das lamelas e regiões centrais da estrutura.

O desvio médio relativo é fator determinante para a escolha do modelo de isoterma que melhor ajustará os dados experimentais, ou seja, o modelo é mais eficiente e adequado ao sistema quando apresentar um menor desvio médio relativo, demonstrando a espontaneidade do processo de adsorção ${ }^{51}$. 
$\mathrm{Na}$ Tabela 3 estão apresentadas as equações propostas para o sistema. É possível observar que as equações que melhor reproduzem os dados experimentais foram aquelas obtidas com o modelo de Langmuir com o desvio de 2,923\% e Temkin (3,574 e 3,758\%) (Figura 6). Os modelos que tiveram desvio padrão na faixa entre 5 a $6 \%$ foram classificados como modelagem matemática satisfatória, acima desta faixa com Freundlich $(15,667 \%)$ indica que a equação não ajustou os dados adequadamente. Fato que pode ser explicado pelos parâmetros n e $K f$ de Freundlich não apresentarem nenhum significado físico e a isoterma formada por este modelo de linearização descreve satisfatoriamente a adsorção do metal dentro de faixa de concentração relativamente baixa, porém acima da faixa de concentração o modelo de Freundlich apresentou limitações, entre elas a irregularidade na condição de contorno adequada da quantidade de íons presentes na estrutura da matriz (parâmetro $K f$ ). Os desvios relativos médios ${ }^{51}$ foram calculados segundo a Equação 6 , sendo n o número de dados experimentais:

$$
100 \times \frac{1}{n} \sum_{n=1}^{n}\left|\frac{N_{f} \exp N_{f} \text { calc. }}{N_{f} \exp }\right|
$$

em que n é o número de dados de adsorção.

Tabela 3. Equações encontradas com a utilização dos modelos de linearização

\begin{tabular}{lccc}
\hline Adsorbato & Modelo & Equação & $\begin{array}{c}\text { Desvio médio } \\
\text { relativo (\%) }\end{array}$ \\
\hline Níquel & Langmuir & $\mathbf{N}_{\mathrm{f}}=10,712 /(1+1,337 \mathrm{C})$ & 5,321 \\
Níquel & Freundlich & $\mathbf{N}_{\mathrm{f}}=4,174 \mathrm{C}^{0,4019}$ & 7,931 \\
Níquel & Temkin & $\mathbf{N}_{\mathrm{f}}=1,193 \ln C+4,564$ & 3,758 \\
Cobre & Langmuir & $\mathbf{N}_{\mathrm{f}}=8,988 /(1+0,913 \mathrm{C})$ & 5,778 \\
Cobre & Freundlich & $\mathbf{N}_{\mathrm{f}}=4,078 \mathrm{C}^{0,415}$ & 5,732 \\
Cobre & Temkin & $\mathbf{N}_{\mathrm{f}}=1,944 \ln C+4,822$ & 3,574 \\
Cobalto & Langmuir & $\mathbf{N}_{\mathrm{f}}=8,246 /(1+0,886 \mathrm{C})$ & 2,923 \\
Cobalto & Freundlich & $\mathbf{N}_{\mathrm{f}}=3,467 \mathrm{C}^{0,464}$ & 15,667 \\
Cobalto & Temkin & $\mathbf{N}_{\mathrm{f}}=1,879 \ln C+4,472$ & 5,321 \\
\hline
\end{tabular}

\section{Termodinâmica de adsorção}

$\mathrm{Na}$ Tabela 4 estão apresentados os resultados dos coeficientes de correlação linear, obtidos a partir das Equações de Langmuir. O modelo de Langmuir apresenta uma vantagem significativa sobre os outros modelos, pois permite quantificar a capacidade de adsorção de íons presentes na estrutura da matriz (b), obter a constante $\mathrm{K}_{\mathrm{L}}$ relacionada com a energia de livre de Gibbs e avaliar a espontaneidade da reação de adsorção.

A termodinâmica do processo de adsorção é definida pelos cálculos dos parâmetros termodinâmicos possibilitados pelo estudo de adsorção, principalmente pelo modelo matemático de Langmuir. A Equação de Langmuir fornece a constante de equilíbrio $\mathrm{K}_{\mathrm{L}}$. Os resultados calorimétricos são aplicados inicialmente à Equação modificada de Langmuir, dada pela expressão $0^{52,53}$ :

$\mathrm{X} / \Delta_{\mathrm{R}} \mathrm{h}=1 /\left(\mathrm{K}_{\mathrm{L}}-1\right) \Delta_{\text {int }} \mathrm{h}+\mathrm{X} / \Delta_{\text {int }} \mathrm{h}$

onde $\mathrm{X}$ é a soma das frações molares de cátions metálicos após a interação com as amostras de esmectita, $\mathrm{K}_{\mathrm{L}}$ é a constante de proporcionalidade que engloba a constante de equilíbrio, $\Delta_{\text {int }} \mathrm{h}$ é a entalpia de interação no equilíbrio do processo e $\Delta_{R}$ h é a entalpia integral de reação.
Para o cálculo da entalpia molar do processo de interação é utilizada a seguinte equação $0^{52,53}$ :

$\Delta \mathrm{H}=\Delta_{\mathrm{int}} \mathrm{h} / \mathrm{N}^{\mathrm{s}}$

sendo $\mathrm{N}^{\mathrm{S}}$ obtido com a batelada pelo experimento de adsorção.

$\mathrm{K}_{\mathrm{L}}$ também favorece o cálculo da energia livre de Gibbs $\left(\Delta_{\text {int }} \mathrm{G}\right)$, que é definida pela expressão $0^{52,53}$ :

$\Delta_{\text {int }} \mathrm{G}=-\mathrm{RT} \ln \mathrm{K}_{\mathrm{L}}$

em que a constante universal dos gases $\mathrm{R}$ é igual a $8,314 \times 10^{-3} \mathrm{kJK}^{-1}$, T é a temperatura (Kelvin).

Uma outra expressão da energia livre de Gibbs está relacionada à $\Delta_{\text {int }} \mathrm{H}$ entalpia e $\Delta_{\text {int }} \mathrm{S}$ entropia, que é definida $\operatorname{por}^{52,53}$.

$\Delta_{\text {int }} \mathrm{G}=\Delta_{\text {int }} \mathrm{H}-\mathrm{T} \Delta_{\text {int }} \mathrm{S}$

Relacionando as duas equações obtem-se $\mathrm{s}^{52,53}$ :

$\ln \mathrm{K}_{\mathrm{L}}=\Delta_{\mathrm{int}} \mathrm{S} / \mathrm{R}-\left(\Delta_{\mathrm{int}} \mathrm{H} / \mathrm{R}\right) \mathrm{T}^{-1}$

Para a esmectita na forma natural e pilarizada foram utilizadas as mesmas soluções de nitrato do experimento de batelada. Na Tabela 4 são expostos os valores das constantes termodinâmicas encontrados com a reação de adsorção. A espontaneidade da reação de adsorção fica evidenciada, com os valores negativos para a energia de livre de Gibbs obtidos com a esmectita na forma original e pilarizada na adsorção dos metais em estudo.

Na Figura 7 está apresentado um exemplo de isoterma de Langmuir obtida com a esmectita pilarizada adsorvendo o cobre cuja regressão linear gera coeficientes angular e linear, que são referentes aos valores de $1 / \Delta_{\text {int }}$ h e $K_{L}$, respectivamente. A constante $K_{L}$, presente na Equação de Langmuir para a calorimetria, é uma constante de proporcionalidade e engloba a constante de equilíbrio ${ }^{52,53}$.

$\mathrm{O}$ valor negativo da entalpia de interação também mostra que a interação é favorável e confere aspectos energeticamente favoráveis desse processo, para o sistema estudado, e o valor positivo da entropia confirma a espontaneidade do processo do ponto de vista energético.

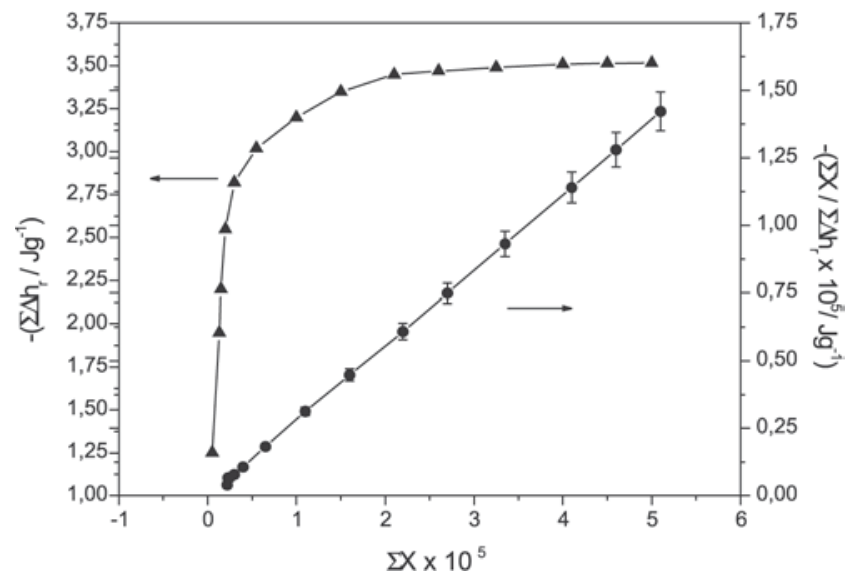

Figura 7. Isoterma de Langmuir $(\mathbf{\Delta})$ e linearização para a esmectita pilarizada (-) (pH 5,0 e temperatura 298,15 $\pm 0,20 \mathrm{~K})$

\section{CONCLUSÕES}

A esmectita originária da Região Amazônica apresentou bom desempenho quanto à recepção de íons intercalantes/pilarizantes em sua estrutura. O processo de pilarização conferiu à esmectita original melhoria 
Tabela 4. Parâmetros termodinâmicos para a adsorção de $\mathrm{Cu}(\mathrm{II}), \mathrm{Ni}(\mathrm{II}), \mathrm{Co}(\mathrm{II})$ (pH 5,0, tempo 360 min, temperatura controlada em $298,15 \pm 0,20 \mathrm{~K})$

\begin{tabular}{lcccccc}
\hline Adsorbato & $\mathrm{N}^{\mathrm{s}}\left(\mathrm{mmol} \mathrm{g}^{-1}\right)$ & $-\Delta_{\mathrm{int}} \mathrm{h}\left(\mathrm{Jg}^{-1}\right)$ & $-\Delta_{\text {int }} \mathrm{H}^{\circ}\left(\mathrm{KJmol}^{-1}\right)$ & $\mathrm{K}_{\mathrm{L}} \times 10^{-3}$ & $-\Delta_{\text {int }} \mathrm{G}^{\mathrm{o}}\left(\mathrm{KJmol}^{-1}\right)$ & $\Delta_{\mathrm{int}} \mathrm{S}^{\circ}\left(\mathrm{JK}^{-1} \mathrm{~mol}^{-1}\right)$ \\
\hline Níquel & 1,03 & 7,14 & $6,93 \pm 0,11$ & 6,81 & $21,88 \pm 0,15$ & $50,18 \pm 0,11$ \\
Cobre & 1,12 & 7,96 & $7,11 \pm 0,11$ & 7,83 & $22,22 \pm 0,15$ & $50,68 \pm 0,11$ \\
Cobalto & 1,45 & 10,56 & $7,30 \pm 0,11$ & 8,91 & $22,55 \pm 0,15$ & $51,15 \pm 0,11$ \\
\hline \multicolumn{7}{c}{$\mathrm{Zr} / \mathrm{Ti}-\mathrm{PILC}$} \\
\hline Níquel & 1,55 & 10,43 & $6,73 \pm 0,11$ & 9,37 & $22,67 \pm 0,15$ & $53,46 \pm 0,11$ \\
Cobre & 1,69 & 11,63 & $6,88 \pm 0,11$ & 10,68 & $22,99 \pm 0,15$ & $54,02 \pm 0,11$ \\
Cobalto & 1,88 & 13,10 & $7,01 \pm 0,11$ & 12,56 & $23,40 \pm 0,15$ & $54,97 \pm 0,11$ \\
\hline
\end{tabular}

substancial em suas propriedades físico-químicas, produzindo material poroso com características favoráveis para a utilização em processos de adsorção com diferentes íons em meio aquoso, caracterizando-se como material potencialmente aplicável no tratamento de efluentes. A afinidade prevaleceu na seguinte ordem para os metais estudados $\mathrm{Cu}>\mathrm{Co}>\mathrm{Ni}$.

Os modelos adotados para a análise das isotermas apresentaram valores bastante próximos daqueles encontrados nas isotermas experimentais, havendo variação maior no modelo de Freundlich. Os resultados encontrados com as equações lineares são bastante semelhantes aos resultados já relatados, situação em que foi utilizado método computacional não linear através do programa "Enzefitte". Observando os resultados de adsorção é possível estabelecer relação entre o número de íons adsorvidos e o número de sítios básicos $(\mathrm{OH})$ produzidos com o processo de pilarização, ou seja, cada sítio básico pertencente à matriz, teoricamente é responsável pelo ancoramento de uma espécie de caráter ácido.

O método de regressão linear adotado neste trabalho não produziu desvio sistemático significativo das isotermas ajustadas, logo, poderá ser potencialmente utilizado com sucesso para descrever processos de troca iônica e adsorção-dessorção relacionados à remoção de íons metálicos presentes em efluentes industriais, como, por exemplo, as indústrias de papel e na extração mineral inadequada envolvendo a problemática da existência indiscriminada de mercúrio em rios.

Para a esmectita original e pilarizada, as interações com os íons dos metais em estudo revelaram-se energeticamente favoráveis. Esse fenômeno foi observado através dos parâmetros termodinâmicos calculados a partir do experimento de batelada e titulações calorimétricas. Esse conjunto de dados permite inferir que o sistema é energeticamente favorável em todos os aspectos, quando da efetivação da interação metal/centros básicos na interface sólido/líquido.

\section{REFERÊNCIAS}

1. Airoldi, C. Em Química de coordenação fundamentos e atualidades; Farias, R. F., ed.; Átomo: São Paulo, 2005, cap. 1.

2. Odendahl, S.; Pulp \& Paper Canada 1994, 95, 30.

3. Buser, H. R.; Environ. Sci. Technol. 1995, 29, 2133.

4. Pontin, J. A.; Massaro, S.; Poluição Química, Ed. Brasiliense: São Paulo, 1993.

5. Fonseca, M. G.; Airoldi, C.; Thermochim. Acta 2000, 1, 359.

6. Pergher, S. B. C.; Corma, A.; Fornes, V.; Quim. Nova 1999, 22, 693.

7. Barrer, R. M.; McLeod, D. M.; Trans. Faraday Soc. 1955, 51, 1290.

8. Vaughan, D. E. W.; Catal. Today 1988, 2, 187.

9. Steat, M.; Patrick, J. W.; Perez, M. J. C.; Water Res. 1995, 29, 467.

10. Takehita, R.; Akimoto, Y.; Nito, S.; Environ. Sci. Technol. 1995, 29, 1186.

11. Boving, T. B.; Wareg, X. J.; Brusseau, M. L.; Environ Sci. Technol. 1999, 33, 764.

12. Heatley, W. R.; Barfield, W. M.; Tappi J. 1995, 78, 197.

13. Terras, C.; Vandervivere, P.; Veastraete, W.; Water Sci. Technol. 1999, 39, 81.

14. Sterte, J.; Burch, R. E.; Catal. Today 1988, 2, 219.

15. Kloprogge, J. T.; Evans. R.; Hickey, L.; Frost, R. L.; Appl. Clay Sci. 2002, $20,157$.
16. Gomes, C. F.; Argilas - o que são e para que servem, Fundação Caloust Gulbenkian: Lisboa, 1988.

17. Moreno, S.; Guitierrez, E.; Alvarez, A.; Papayannakos, N. G.; Poncelet, G.; Appl. Catal. 1997, 165, 103.

18. Valverde, J. L.; Sanchez, P.; Dourado, F.; Molina, C. B.; Romero. A.; Microporous Mesoporous Mater. 2002, 54, 155.

19. Ooka, C.; Yoshida, H.; Horio, M.; Suzuki, K.; Hattori, T.; Appl. Catal. 2003, $41,313$.

20. Guerra, D. L.; Lemos, V. P.; Angélica, R. S.; Airoldi, C.; Rev. Soc. Port. Mater. 2006, 17, 75.

21. Guerra, D. L.; Lemos, V. P.; Angélica, R. S.; Airoldi, C.; Polyhedron 2006, $15,2880$.

22. Volzone, C.; Microporous Mesoporous Mater. 2001, 49, 197.

23. Sychev, M.; Rozwadowski, M.; Sommen, A. P. B.; Beer, V. H. J.; van Santen, R. A; Microporous Mesoporous Mater. 2004, 37, 187.

24. Santos, P. S.; Ciência e Tecnologia de Argilas, 2a ed., Edgard Blücher: São Paulo, 1989.

25. Mott, C. J. B.; Catal. Today1988, 2, 199.

26. Pinnavaia, T. J.; Science 1983, 220, 365.

27. Fonseca, M. G.; Oliveira, A. S.; Airoldi, C.; J. Colloid Interface Sci. 2001, $240,533$.

28. Luna, J. F.; Schuchardt, U.; Quim. Nova 1999, 22, 104

29. Occelli, M. L.; Hsu, J. T.; Galya, L. G.; J. Mol. Catal. A: Chem. 1985, 33, 371.

30. Cañizares, P.; Valverde, J. L.; Sun Kou, M. R.; Molina, C. B.; Microporous Mesoporous Mater. 1999, 29, 267.

31. Airoldi, C.; Farias, R. F.; Quim. Nova 2000, 23, 496.

32. Fonseca, M. G.; Airoldi, C.; Quim. Nova 2003, 23, 699

33. Silva, C. R.; Jardim, I. C. S. F.; Airoldi, C.; J. Sep. Sci. 2001, 24, 197.

34. Arakaki, L. N. H.; Airoldi, C.; Polyhedron 2000, 19, 367.

35. Brandt, K. B.; Kydd, R. A.; Appl. Catal. 1997, 165, 327.

36. Hutson, N. D.; Hoesksta, M. J. ;Yang, R. T.; Microporous Mesoporous Mater. 1999, 28, 267.

37. Occelli, M. L.; Tindwa, R. M.; Clays Clay Miner. 1983, 32, 22.

38. Bahamond, A.; Mohino, F.; Rebolar, M.; Yates, M.; Avila, P.; Mendioroz, S.; Catal. Today 2001, 69, 233.

39. Bottero, J. Y.; Axelos, M.; Tchoubar, D.; Cases, J. M.; Fripiat, J. J.; Fiessinger, F.; J. Colloid Interface Sci. 1987, 47, 117.

40. Zhao, D.; Chem. Mater. 1993, 4, 1433.

41. Bayley, S. W; Brindley G. W.; Brown G.; Structures of layer silicates. Mineralogical society, Monograph, Cristal strutures of clay minerals and their X-ray identification, Mineralogical Society: London, 1980.

42. Blindley, G. W.; Samples R. E.; Clays Clay Miner. 1997, 12, 229.

43. Livage, J. Em Proceeding of the Winter School on Glasses and Ceramics from gels; Science and Tecnology, Aegerter, M. A.; Jafelicci Jr., M. Souza, D. F.; Zanotto, E. D., eds.; Universidade Federal de São Carlos: São Paulo, 1989.

44. Arakaki, L. N. H.; Airoldi, C.; Quim. Nova 1999, 22, 246.

45. Airoldi, C.; Nunes, L. M. ; Farias, R. F.; Mater. Res. Bull. 2000, 35, 2081.

46. Nunes, L. M. ; Airoldi, C.; Chem. Mater. 1999, 11, 2069.47.

Santos, R. V.; de Carvalho Jr., O. A.; Martins, E. S. Em Caracterização Geoquímica de Águas e Sedimentos de Corrente do Distrito Federal, Relatório Final de Atividades; Boaventura, G. R., coord.; FAPDF/UnB/IG: Brasília, 1997.

48. Atkins, P. W.; Physical Chemistry, $5^{\text {th }}$ ed., Oxford University Press: Oxford, 1994.

49. Harter, R.; Soil Sci. Soc. Am. J. 1984, 48, 749

50. Barrow, N. J.; Soil Sci. 1978, 29, 447.

51. Sodré, F. F.; Peralta-Zamora, P. G.; Grassi, M. T.; Quim. Nova 2004, 27, 695

52. Sales, J. A. A.; Airoldi, C.; Thermochim. Acta 2005, 77, 427.

53. Sales, J. A. A.; Airoldi, C.; J. Non-Cryst. Solids 2003, 142, 330. 